$09.2 ; 11.3 ; 14$

\title{
Полностью оптический магнитометрический датчик для задач магнитоэнцефалографии и томографии сверхслабого поля
}

\author{
(C) А.К. Вершовский, А.С. Пазгалев, М.В. Петренко \\ Физико-технический институт им. А.Ф. Иофффе РАН, Санкт-Петербург, Россия \\ E-mail: antver@mail.ioffe.ru
}

Поступило в Редакцию 15 апреля 2020г.

В окончательной редакции 23 мая 2020г.

Принято к публикации 1 июня 2020г.

\begin{abstract}
Предложен и экспериментально исследован вариант схемы магнитометрического датчика на атомарных парах цезия, использующий возбуждение магнитного резонанса модулированным излучением поперечной по отношению к магнитному полю сверхтонкой оптической накачки. Показано, что при использовании ячейки объемом $0.125 \mathrm{~cm}^{3}$ вариационная чувствительность такой схемы, оцененная по отношению крутизны сигнала в центре магнитного резонанса к дробовым шумам детектирующего излучения, достигает уровня $<10 \mathrm{fT} / \mathrm{Hz}^{1 / 2}$ в полосе частот порядка $850 \mathrm{~Hz}$. Датчик, не излучающий радиочастотные поля, предназначен для работы в магнитоэнцефалографических комплексах. Рассмотрены возможные способы повышения быстродействия схемы для детектирования относительно быстрых ( $4.2 \mathrm{kHz}$ в поле $0.1 \mathrm{mT})$ сигналов прецессии магнитных моментов протонов в перспективных схемах томографии сверхслабого поля.
\end{abstract}

Ключевые слова: оптически детектируемый магнитный резонанс, квантовый магнитометр, магнитоэнцефалография, томография сверхслабого поля.

DOI: 10.21883/PJTF.2020.17.49894.18340

Решение глобальной задачи исследования, интерпретации и моделирования принципов работы головного мозга, стоящей сегодня перед человечеством, невозможно без методик магнитоэнцефалографии (МЭГ) [1] и магнитно-резонансной томографии (МРТ) [2]. Хотя среди сканирующих систем МРТ доминируют системы с напряженностью поля от 1 до $3 \mathrm{~T}$, в последние годы активно разрабатываются системы МРТ сверхслабого поля (ССП), в которых ориентация магнитных моментов протонов производится полем порядка $100 \mathrm{mT}$, а их прецессия регистрируется в поле порядка $0.1 \mathrm{mT}$ [3]. Важным фактором, способствующим разработке схем МРТ ССП, является потенциальная возможность их объединения с системами МЭГ. Основную трудность при реализации схем МРТ ССП представляет регистрация сигнала: в поле $0.1 \mathrm{mT}$ частота прецессии протонов составляет примерно $4.2 \mathrm{kHz}$, что исключает возможность использования индукционных датчиков. Существующие решения этой проблемы [4] предполагают использование сверхпроводящих датчиков СКВИД (SQUID), чувствительность которых не падает с частотой. Однако датчикам СКВИД свойственны ограничения на величину перепадов измеряемых полей, что затрудняет их использование в импульсных системах [5]. Кроме того, их обслуживание крайне дорого, а соответствующие системы громоздки, что усложняет создание передвижных диагностических систем. Единственный класс датчиков, который сегодня способен удовлетворить требованиям объединенных систем МЭГ-МРТ ССП - это квантовый магнитометр с оптической накачкой (КМОН) [6,7].

В последние годы развитие малогабаритных высокочувствительных КМОН, предназначенных для биоме- дицинских приложений, идет по двум направлениям: создание магнитометров, работающих в режиме SERF (spin-exchange relaxation free) - подавление спинобменного уширения линии магнитного резонанса (MP) в нулевом магнитном поле [8,9], и альтернативных им схем КМОН, работающих в режиме светового подавления спин-обменного уширения $[10,11]$. Схемы SERF характеризуются более высокой предельной чувствительностью, но их применение ограничено нулевыми полями, и хотя уже были предложены способы их применения в системах МРТ ССП (такие, как передача сигнала МРТ по трансформаторной линии в магнитоэкранированный объем к датчику SERF [12]), КМOH ненулевого поля пока что остаются единственными кандидатами на использование непосредственно в схемах МРТ ССП.

Разработка многоканальных систем МЭГ-МРТ ССП, базирующихся на таких датчиках, требует решения двух основных проблем: 1) устранение взаимовлияния соседних датчиков, генерирующих резонансные радиочастотные поля с близкими частотами; 2) расширение полосы чувствительности. В настоящей работе приводятся результаты исследования схемы датчика, которая потенциально позволяет решить указанные проблемы и при этом характеризуется необходимой чувствительностью.

В предлагаемой схеме метод возбуждения МР поперечным по отношению к магнитному полю лучом, модулированным на частоте прецессии магнитных моментов (ларморовской частоте), предложенный в [13], применен к схеме накачки, в которой световое подавление спин-обменного уширения МР на сверхтонком уровне $F=I+1 / 2$ (здесь $I-$ момент ядра, для Cs $I=7 / 2$ ) 


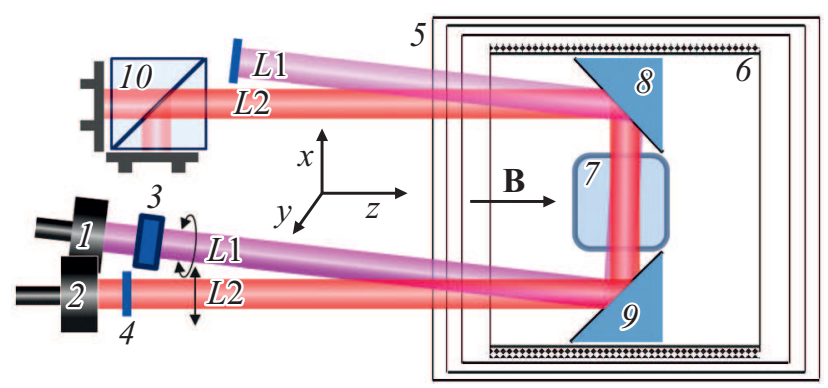

Рис. 1. Упрощенная схема квантового датчика. $1-$ ввод излучения накачки, $2-$ ввод пробного излучения, $L 1-$ луч оптической накачки, $L 2-$ пробный луч, 3 - электрооптический (циркулярный) модулятор поляризации, 4 - линейный поляризатор, 5 - магнитный экран, 6 - соленоид, 7 газовая ячейка с парами $\mathrm{Cs}, 8,9-$ зеркала, 10 - балансный фотоприемник.

реализуется при монохроматической лазерной накачке, настроенной в резонанс с переходом со сверхтонкого уровня $F=I-1 / 2$ [14]. Детектирование МР осуществляется на ларморовской частоте (так называемая $M_{X}$-схема) по повороту поляризации нерезонансного линейно поляризованного поперечного пробного луча.

Экспериментальная установка, на которой проводились измерения, описана в $[15,16]$. Были использованы полупроводниковые лазеры с внешними резонаторами VitaWave ECDL-8920R. Стеклянная кубическая ячейка производства компании VitaWave размером $5 \times 5 \times 5 \mathrm{~mm}$, содержащая насыщенные пары Cs и азот $\left(\mathrm{N}_{2}\right)$ под давлением $\sim 100$ Torr, была расположена в многослойном магнитном экране, индукция поля в котором поддерживалась на уровне $12 \mu \mathrm{T}$ (рис. 1). Установка была дополнена электрооптическим модулятором Thorlabs EO-AM-NR-C1, позволяющим переключать направление круговой поляризации луча накачки; частота переключения сканировалась в окрестности ларморовской частоты $\mathrm{Cs}(42 \mathrm{kHz})$. Луч накачки был направлен перпендикулярно магнитному полю под небольшим углом к пробному лучу.

Амплитуда и ширина МР были исследованы при различных температурах ячейки и интенсивностях излучения накачки. На основе этих значений и интенсивности пробного луча на балансном фотоприемнике согласно $[17,18]$ оценивалась предельная вариационная чувствительность датчика, ограниченная дробовым шумом фототока.

Результаты измерений приведены на рис. 2. Видно, что зависимость ширины линии МР Г от интенсивности накачки условно можно разделить на три области: область преобладания спин-обменного уширения, в которой ширина полностью определяется температурой ячейки; область частичного подавления спин-обменного уширения МР светом накачки; область преобладания светового уширения МР. В нашем эксперименте снижение ширины МР во второй области не превышает 25\%, тогда как в пределе оно может достигать нескольких раз [19]. Это обусловлено, в частности, недостаточной отстройкой частоты пробного излучения, связанной с техническими ограничениями, которая привела к дополнительному уширению резонанса примерно на $300 \mathrm{~Hz}$ (HWHM). Несмотря на это, благодаря высокому соотношению сигнал/шум, достигающему $157 \mathrm{~dB}$ в полосе $1 \mathrm{~Hz}$, вариационная чувствительность такой схемы, оцененная по отношению крутизны сигнала в центре МР к дробовым шумам детектирующего излучения, достигает уровня $<10 \mathrm{fT} / \mathrm{Hz}^{1 / 2}$.

Здесь необходимо отметить, что, во-первых, чувствительность такого датчика также дополнительно ограничена квантовым проекционным шумом, что при оптимальных параметрах приводит к снижению чувствительности примерно в $\sqrt{ } 2$ раза $[16]$; во-вторых, рассматриваемая схема оказывается более чувствительной к шумам излучения накачки, чем стандартная схема. Это обусловлено тем, что в ней МР возбуждается модуляцией параметров накачки, и любые вариации этих параметров в окрестности частоты MP (а также ее субгармоник) преобразуются в шум в канале регистрации. Оценки показывают, что для реализации предельной чувствительности схемы технические шумы излучения накачки на этих частотах должны быть подавлены до уровня, превышающего уровень дробового шума не более чем в 2-3 раза (так, в нашей схеме примерно десятикратное превышение шума интенсивности излучения накачки
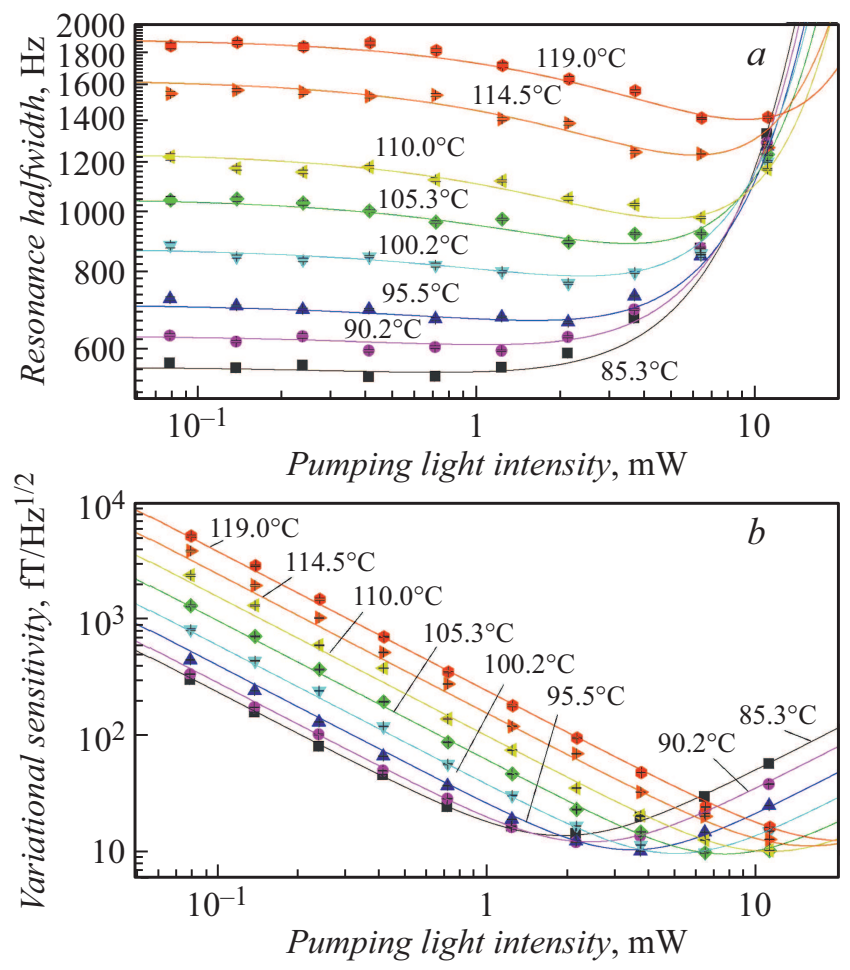

Рис. 2. Зависимости ширины магнитного резонанса (НWHM) $(a)$ и предельной реализуемой чувствительности $(b)$ от интенсивности излучения накачки при различных температурах ячейки. 


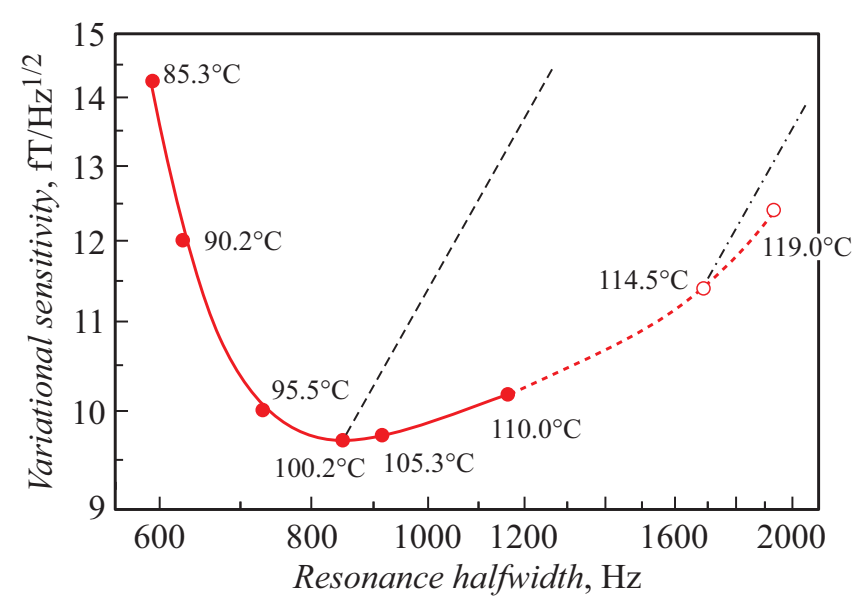

Рис. 3. Параметрическая зависимость предельной реализуемой чувствительности от ширины резонанса. Числа соответствуют температурам, при которых эта чувствительность реализуется. Последние две точки - результат экстраполяции кривых, приведенных на рис. $2, b$.

над дробовым шумом приводило к двух-трехкратному ухудшению чувствительности).

В схемах МЭГ для передачи излучения накачки в датчик можно использовать поддерживающее линейную поляризацию оптоволокно; такие волокна имеют две собственные моды, направления азимута поляризации для которых различаются на $90^{\circ}$. Соответствующее переключение линейной поляризации излучения на входе в оптоволокно будет преобразовано четвертьволновой пластинкой в переключение знака циркулярной поляризации непосредственно в датчике. Требуемое подавление шумов может быть осуществлено методами активной стабилизации интенсивности лазерного излучения, измеряемой на выходе оптоволокна.

На рис. 3 приведена параметрическая зависимость предельной реализуемой чувствительности от Г и указаны температуры, при которых эта чувствительность реализуется. Этот рисунок иллюстрирует связь между чувствительностью и быстродействием датчика. Наилучшая чувствительность $\delta B=9.7 \mathrm{fT} / \mathrm{Hz}^{1 / 2}$ реализуется при $\Gamma \approx 850 \mathrm{~Hz}$; эти параметры позволяют успешно использовать такую схему в системах МЭГ, сигналы в которых обычно лежат в диапазоне частот $2-200 \mathrm{~Hz}$.

Как известно [20], $M_{X}$-датчики способны детектировать сколь угодно быстрые вариации магнитного поля, в том числе и те, частота $f$ которых превышает $Г$, но отношение сигнал/шум при регистрации таких сигналов падает пропорционально $1 / f$, т.е. амплитудночастотная характеристика отклика МР в схеме $M_{X}$-датчика похожа на амплитудно-частотную характеристику фильтра низких частот первого порядка с частотой среза, равной Г. Этот факт на рис. 3 проиллюстрирован линиями, показывающими, как вариационная чувствительность меняется при превышении $f$ над шириной MP при прочих фиксированных параметрах. Из рисунка следует, что увеличение полосы до $\sim 1700 \mathrm{~Hz}$ достижимо без существенных потерь чувствительности (точка $T=114.5^{\circ} \mathrm{C}$ ). Дальнейшее повышение температуры не оправдано, так как чувствительность начинает ухудшаться со скоростью, близкой к $1 / f$. Кроме того, как следует из рис. 2, высокие температуры требуют интенсивной накачки. Дальнейшее увеличение $f$ при прочих фиксированных параметрах приводит к спаду чувствительности $1 / f$. На частоте $4.2 \mathrm{kHz}$ достижимая чувствительность ухудшается примерно в 2.5 раза до уровня $\sim 30 \mathrm{fT} / \mathrm{Hz}^{1 / 2}$ (штрихпунктирная линия на рис. 3). Сочетание этих параметров позволяет рассчитывать также и на успешное использование таких датчиков в схемах МРТ ССП, в том числе и в объединенных с системами МЭГ.

Таким образом, нами исследована схема магнитометрического датчика, предназначенного для работы в ненулевых полях и не использующего радиочастотные поля. Показано, что она потенциально способна обеспечить уникальное сочетание чувствительности и быстродействия, требуемых в магнитоэнцефалографии и магнитнорезонансной томографии сверхслабого поля. Наиболее сложным, но преодолимым препятствием на пути к созданию такого датчика является требование подавления шумов излучения лазерной накачки до уровня, близкого к уровню дробового шума.

\section{Финансирование работы}

Работа выполнена при финансовой поддержке Российского фонда фундаментальных исследований в рамках научного проекта № 19-29-10004.

\section{Конфликт интересов}

Авторы заявляют, что у них нет конфликта интересов.

\section{Список литературы}

[1] Hämäläinen M., Hari R., Ilmoniemi R.J., Knuutila J., Lounasmaa O.V. // Rev. Mod. Phys. 1993. V. 65. N 2. P. 413 497. DOI: 10.1103/RevModPhys.65.413

[2] Van der Zwaag W., Schäfer A., Marques J.P., Turner R., Trampel R. // NMR Biomed. 2016. V. 29. N 9. P. 1274-1288. DOI: $10.1002 / \mathrm{nbm} .3275$

[3] Coffey A.M., Truong M.L., Chekmenev E.Y. // J. Magn. Reson. 2013. V. 237. P. 169-174. DOI: 10.1016/j.jmr.2013.10.013

[4] Inglis B., Buckenmaier K., SanGiorgio P., Pedersen A., Nichols M.A., Clarke J. // Proc. Natl. Acad. Sci. USA. 2013. V. 110. N 48. P. 19194. DOI: 10.1073/pnas.1319334110

[5] Parkkonen L., Ilmoniemi R.J., Lin F.-H., Espy M. Ultra-low-field MRI and its combination with MEG // Magnetoencephalography: from signals to dynamic cortical networks / Eds S. Supek, C.J. Aine. Berlin-Heidelberg: Springer, 2014. P. 941-972.

[6] Вершовский А.К., Пазгалев А.С. // ЖТФ. 2008. Т. 78. В. 5. С. 116-124. [Пер. версия: 10.1134/S1063784208050198]. 
[7] Borna A., Carter T.R., Goldberg J.D., Colombo A.P., Jau Y.-Y., McKay J., Weisend M., Taulu S., Stephen J.M., Schwindt P.D.D. // Phys. Med. Biol. 2017. V. 62. N 23. P. 8909-8923. DOI: 10.1088/1361-6560/aa93d1

[8] Kominis I.K., Kornack T.W., Allred J.C., Romalis M.V. // Nature. 2003. V. 422. N 6932. P. 596-599.

DOI: $10.1038 /$ nature 01484

[9] Dang H.B., Maloof A.C., Romalis M.V. // Appl. Phys. Lett. 2010. V. 97. N 15. P. 151110. DOI: $10.1063 / 1.3491215$

[10] Scholtes T., Schultze V., IJsselsteijn R., Woetzel S., Meyer H.-G. // Phys. Rev. A. 2011. V. 84. N 4. P. 043416. DOI: $10.1103 /$ PhysRevA.84.043416

[11] Schultze V., Schillig B., IJsselsteijn R., Scholtes T., Woetzel S., Stolz R. // Sensors. 2017. V. 17. N 3. P. 561. DOI: $10.3390 / \mathrm{s} 17030561$

[12] Savukov I.M., Stolz V.S., Volegov P.L., Espy M.A., Matlashov A.N., Gomez J.J., Kraus R.H. // J. Magn. Reson. 2009. V. 199. N 2. P. $188-191$. DOI: $10.1016 /$ j.jmr.2009.04.012

[13] Bell W.E., Bloom A.L. // Phys. Rev. Lett. 1961. V. 6. N 6. P. 280-281. DOI: 10.1103/PhysRevLett.6.280

[14] Попов Е.Н., Бобрикова В.А., Воскобойников С.П., Баранцев К.А., Устинов С.М., Литвинов А.Н., Вершовский А.К., Дмитриев С.П., Картошкин В.А., Пазгалев А.С., Петренко М.В. // Письма в ЖЭТФ. 2018. Т. 108. В. 8. С. 543548. [Пер. версия: 10.1134/S0021364018200122].

[15] Ossadtchi A.E., Kulachenkov N.K., Chuchelov D.S., Dmitriev S.P., Pazgalev A.S., Petrenko M.V., Vershovskii A.K. // 2018 Int. Conf. Laser Optics (ICLO). IEEE, 2018. P. 543. DOI: 10.1109/LO.2018.8435740

[16] Вершовский А.К., Дмитриев С.П., Козлов Г.Г., Пазгалев А.С., Петренко М.В. // ЖТФ. 2020. Т. 80. В. 8. С. $1243-$ 1253. DOI: $10.21883 /$ JTF.2020.08.49533.438-19

[17] Alexandrov E.B., Balabas M.V., Pasgalev A.S., Vershovskii A.K., Yakobson N.N. // Laser Phys. 1996. V. 6. N 2. P. 244-251.

[18] Budker D., Romalis M. // Nature Phys. 2007. V. 3. N 4. P. 227-234. DOI: $10.1038 /$ nphys566

[19] Jau Y.-Y., Post A.B., Kuzma N.N., Broun A.M., Romalis M.W.V., Happer W. // Phys. Rev. Lett. 2004. V. 92. N 11. P. 110801. DOI: 10.1103/PhysRevLett.92.110801

[20] Groeger S., Bison G., Schenker J.-L., Wynands R., Weis A. // Eur. Phys. J. D. 2006. V. 38. N 2. P. 239-247.

DOI: $10.1140 /$ epjd/e2006-00037-y 